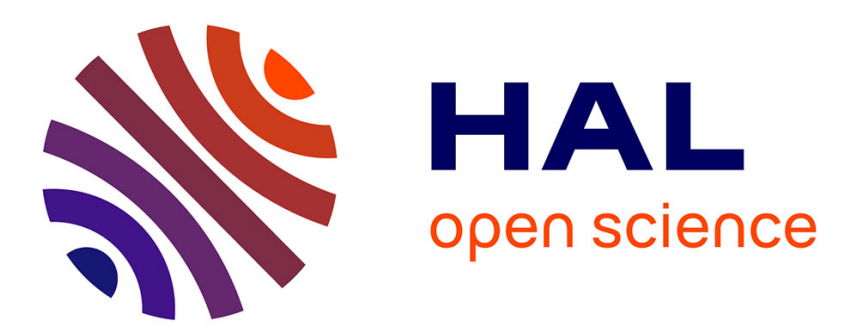

\title{
A human-machine cooperative approach for time series data interpretation
}

Thomas Guyet, Catherine Garbay, Dojat Michel

\section{To cite this version:}

Thomas Guyet, Catherine Garbay, Dojat Michel. A human-machine cooperative approach for time series data interpretation. 11th Conference on Artificial Intelligence in Medicine (AIME 2007), Jul 2007, Amsterdam, Netherlands. pp.3-12. inserm-00519815

\section{HAL Id: inserm-00519815 https://www.hal.inserm.fr/inserm-00519815}

Submitted on 8 Jun 2011

HAL is a multi-disciplinary open access archive for the deposit and dissemination of scientific research documents, whether they are published or not. The documents may come from teaching and research institutions in France or abroad, or from public or private research centers.
L'archive ouverte pluridisciplinaire $\mathbf{H A L}$, est destinée au dépôt et à la diffusion de documents scientifiques de niveau recherche, publiés ou non, émanant des établissements d'enseignement et de recherche français ou étrangers, des laboratoires publics ou privés. 


\title{
A Human-Machine Cooperative Approach for Time Series Data Interpretation
}

\author{
Thomas Guyet ${ }^{1}$, Catherine Garbay ${ }^{2}$, and Michel Dojat ${ }^{3}$ \\ 1 TIMC Laboratory, Domaine de la Merci F-38706 La Tronche, France \\ Thomas.Guyet@imag.fr \\ ${ }^{2}$ CNRS/LIG Laboratoire d'Informatique de Grenoble, France \\ ${ }^{3}$ UMR-S 836 Inserm/UJF/CEA "Institut des Neurosciences", Grenoble, France
}

\begin{abstract}
This paper deals with the interpretation of biomedical multivariate time series for extracting typical scenarios. This task is known to be difficult, due to the temporal nature of the data at hand, and to the context-sensitive aspect of data interpretation, which hamper the formulation of a priori knowledge about the kind of patterns to detect and their interrelations. A new way to tackle this problem is proposed, based on a collaborative approach between a human and a machine by means of specific annotations. Two grounding principles, namely autonomy and knowledge discovery, support the co-construction of successive abstraction levels for data interpretation. A multi-agent system is proposed to implement effectively these two principles. Respiratory time series data (Flow, Paw) have been explored with our system for patient/ventilator asynchronies characterization studies.
\end{abstract}

\section{Introduction}

In the mass of monitoring data, the detection of specific patterns relative to a significant event requires a time-consuming visual data examination by an expert. The relations between events occurring in various physiological parameters are meaningful but difficult to identify. In addition, the potential generic character of the detected patterns is rarely pointed out. We assume that without the use of a computerized assistant, this mass of data can not be fully exploited. However, the design of such an assistant is difficult because the temporal and multivariate nature of the data at hand and the context-sensitive aspect of data interpretation hamper the formulation of a priori knowledge about the kind of patterns to detect and their interrelations. The number of patterns and their combination to form meaningful situations is potentially very high, making their systematic enumeration and description by a human impossible.

For instance, in the context of patients hospitalized in Intensive Care Unit (ICU) and mechanically ventilated, asynchrony, i.e. a frequent mismatch between the ventilator assistance and the patient demand, has recently been reported [1]. Automatic asynchrony detection could be used to adjust ventilator settings and then improve mechanical assistance efficiency. However, to model asynchrony patterns for a robust automatic detection, essential information is still missing.

R. Bellazzi, A. Abu-Hanna, and J. Hunter (Eds.): AIME 2007, LNAI 4594, pp. 3-12 2007.

(C) Springer-Verlag Berlin Heidelberg 2007 
In this paper, we propose a collaborative human-machine approach to gradually define from the data the significant patterns to detect, and to discover specific temporal relations between events.

Following [2], we advocate the use of annotations as an elegant and efficient way for communicating between a human and a machine. Starting from expert annotations, the computer builds its own abstractions and in turn annotates the signals. The human-machine interactions occur at three abstraction levels (e.g. segmented time series, symbolic time series data and scenarios), around which a collaborative approach to the interpretation of time series data is proposed. We implemented our approach using the multi-agent (MA) paradigm. The MA system (MAS) design is based on two grounding principles, namely autonomy and knowledge discovery, which support the construction by the system of data interpretation at successive abstraction levels.

\section{State of the Art}

Knowledge-Based Temporal Abstraction. Basically, abstraction transforms initial numerical time series to symbolic time series, which is known to result in a so-called semantic gap. The larger is the gap the more difficult is the abstraction. To fill this gap, as showed by Shahar [3], the abstraction process relies on several mechanisms and uses various types of a priori knowledge. There is a wide literature regarding the way to realize several temporal abstraction levels in medical domains 4 .

For instance, in the Calicot system [5], dedicated to cardiac arrhythmias detection, a priori knowledge is introduced to support the discovering of new rules. Neural networks are trained to discover normal or abnormal P-waves and QRScomplexes and translate physiological time series into symbolic time series. Then, the system learns rules with an Inductive Logic Programming method where $a$ priori knowledge and bias can be introduced. The computed rules show complex temporal relations between the symbols attached to the various arrhythmias, which are difficult to understand by an expert. However, ICU data appears unfortunately insufficiently formalized to envisage such a procedure.

Guimarães et al. [6] propose the TCon method for automated temporal knowledge acquisition applied to sleep-related respiratory disorders. TCon uses machine learning techniques to discover new elementary patterns (rules) from the time series. These rules are then translated, in order to be easily interpretable for the clinician, a central motivation that we share. But we are then facing a trade-off: too much a priori knowledge hampers knowledge discovery, whilst not enough a priori knowledge produces non-coherent or difficult to interpret abstractions. Step by step validation of the abstraction process by the human expert, based on well-adapted visual representations, has been shown as a way to build robust abstraction process [7. In the same vein, we propose that during the knowledge discovery process, the expert continuously interacts with the learning system to drive the process and extract useful chunks of knowledge. 
Collaborative Knowledge Discovery. The development of mutual learning capabilities is a way to solve the previously mentioned trade-off between autonomy and intelligibility. In [8, the authors present the desired cycle of knowledge where clinicians are central actors in knowledge production with a positive impact on the final system. Classical approaches to collaborative knowledge discovery consist either in human-driven machine learning, the clinician acting as an expert, or in computer assisted human learning, the machine acting as a teacher (Intelligent Tutoring Systems). TeachMed [9] for instance is a recent system to support clinical learning. The student is observed and when, for TeachMed, he/she needs assistance a dialog is engaged to help him/her to find the solution. Computers can also serve as a medium for a collaborative mutual learning (Computer-Supported Collaborative Learning) in facilitating interaction between humans 10. In 11, human and machine are both embedded in a learning cycle. On this basis, they automatically generate in a faster way more reliable guidelines compared to classical methods.

Multi-agent systems exhibit several properties that make them a "natural" framework for the design of such collaborative systems, particularly due to the open character of the MA architecture and to the autonomy of its components. They facilitate the combination of several approaches for distributed problem solving, support the autonomous emergence of new knowledge and may naturally integrate a human user as a specific agent. According to this view, the MASKS environment 12 introduces agent interactions based on cooperative behaviors to facilitate knowledge discovery. Agents, that encapsulated machine learning algorithms, respectively evaluate and may merge their generated rules. In [13], a MAS is used to collaboratively annotate artistic artifacts. The system opens a share space to enable experts to insert their own annotations and learn mutually from each other.

Interactive knowledge construction literature indicates that human/machine interaction enables knowledge discovery both for the machine and the human. Whilst the human helps to focus the machine learning process to the discovery of useful chunk of knowledge, the machine helps to focus the human learning process to a deep exploration of the data at hand.

\section{The Methodological Concepts}

Signal Interpretation. We consider interpretation as a complex process involving complementary exploration, description and annotation tasks, which are operated by a collection of autonomous agents (including humans), working to progressively refine the interpretation [13. The role of the exploration task is to focus the attention, at each abstraction level, on relevant information, in terms of time series segments, event classes or typical event relations. In our system, several steps are necessary before reaching the upper notion of scenario: 1) Segmentation, 2) Symbolic time series and 3) Scenario construction. The description and annotation steps are situated in a dynamic context which includes the agent past experience and additional information on data. The description task aims 
to build numerical and symbolical models of the information under interest, possibly by fusion. Finally, the annotation task role is to attach symbolic labels to newly provided raw data using the constructed models.

Human-Machine Collaboration. We propose to approach the interpretation of time series data as performed by two cooperating agents - a man and a machine - operating across three successive abstraction levels (segments, symbolic time series and scenarios). These agents share a common environment (e.g. annotations, time series data, segments ...) and mutually interact via annotations to progressively refine their own interpretation. In the absence of consistent $a$ priori knowledge and considering the difficulty of this processing, an active partnership between man and machine is sought. It does not come down to a fixed request-answer interaction scheme in which each actor is meant to compensate for the lack of knowledge of its partner, and therefore supposed to share its world of meaning. This partnership is rather meant to allow a co-construction of annotations, in which the interpretation of facts is not defined beforehand by one of the actors, but co-constructed in the course of their interaction. According to this principle, each partner is in turn given the possibility to observe and interpret annotations provided by its partner, and/or to propose annotations judged as appropriate according to a given interpretation context. In such framework, each partner is meant to "play" in its own world of meaning, thus preserving its autonomy, with no prevalence of one on the other. In consequence, there is a possibility of learning and discovery for both partners. To be noticed is the fact that any information - be it a case example or a conceptual interpretation is provided within its context of appearance, so that interaction develops in a situated way.

\section{A Multi-Agent System for Time Series Interpretation}

Central to our design, is the distinction between three main tasks, namely segmentation (localization of relevant segments), classification (symbolic labeling of the segments), and scenario construction (computation of inter-symbol relationships). Dedicated agents are conceived to support these various tasks; they make use of specific models, which drive the way to explore and describe, via annotations, the information at hand (forward application). These models are autonomously computed (backward application), based on knowledge discovery methods, and according to previously computed annotations, which may be provided by an external user, or by other agents. Interaction and feedback loops occur all along the interpretation process to ensure its progressive refinement.

To be noticed is the fact that all these processes, as the human agent himself, run in parallel and may interact at any time, thanks to the annotation process which results in modification propagated within the information at hand, and thus altering but not disrupting the interpretation process. 


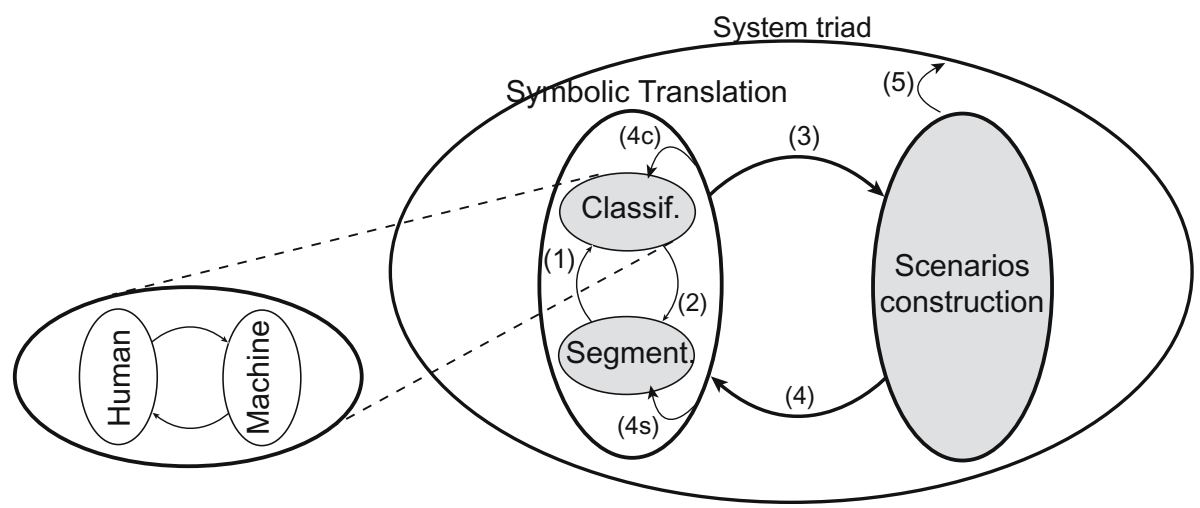

Fig. 1. MAS entities organization according to recursive triads. The classification triad is expanded to show the man-machine interaction.

\subsection{System Design}

A global view of the system architecture is presented in Figure 1, A triadic organization is proposed as a conceptual framework to support the needed inter-agent interactions and feedback loops. A triad is made of two processing entities working in reciprocal interaction that constitutes a more abstract processing whole. Each abstraction level is organized into a triad, giving rise to new annotation elements to be processed at a higher abstraction level (upper triad). Conversely, each upper level triad may launch lower-level triadic agents (feedback loop). Time series data are processed within the segmentation and classification triads, which mutually interact (links 1 and 2, Figure1) within the symbolic translation triad to build symbolic time series. Symbolic time series are processed within the scenario construction triad. The symbolic translation and scenario construction triads mutually interact within the system triad (links 3 and 4). Scenarios are the final outcome to the system triad (link 5). When entering a feedback loop (link 4, 4s and 4c), the constructed scenarios may conversely be used to propagate modifications to the symbolic time series, and from this triad to the lower level classification and segmentation agents.

\subsection{Agentification}

Classification Agents. For the sake of simplification, we first of all describe the system style of working in front of univariate time series. The general control cycle of a classification agent is (i) to collect segments (ii) to compute segment descriptors (iii) to classify them and (iv) to build segment class models. The segments to analyze may be pointed out by a human, or delimited by the segmentation agents. At system start, some initial annotations or models are provided to some agent, to launch the whole interpretation process.

Each segment is described by a feature vector (typically 20 sampled values and the length of the segment), which are classified. A weighted Euclidean 
distance is used to compare them. The $k$-mean algorithm has been selected because of its capacity to work under our specific constraints: lack of a priori knowledge (in particular no a priori knowledge of the number of classes to observe), and dynamicity (evolution of the class characterization along the interpretation in progress). We use an extended version of the algorithm, in which different distances (i.e. adaptive weights in the Euclidean computation) are selected and computed, depending on the class under interest. For each class, the mean vector and the distances weights constitute the model of the segments of this class, attached to a symbolic name with no proper semantic.

The next issue to consider is then how to match this symbolic name with an intelligible denomination for the clinician (e.g. asynchrony events). This is performed by means of triadic man-machine interaction, according to which any modification (e.g. annotation) performed by any agent is merely a perturbation of the information under interpretation. According to this view, the system interpretation may be shifted toward human interpretation in a transparent way.

Segmentation Agents. For each class of segments constructed by the classification agent, a new segmentation agent is created, and provided with the corresponding segment model. Consequently, the number of segmentation agents changes in the course of the interpretation. Each segmentation agent performs a pattern matching algorithm : It calculates the distance between the agent's segment model and a sliding window of variable length, that delineates a segment, moving on the time series. Then, the segments closest to the model are selected. A non-overlapping constraint is used to eliminate further segment hypotheses. Distances are computed in the model parameter space (the previously described vectorial description of a segment), rather than in the time series space, to cope with differences of pattern size. The segmentation of a time series is the concatenation of the segments constructed by all these agents.

Here again, annotations are exchanged between the machine and the human via the interface. Segments are shown to the human through the system visualization interface. Such visualization allows focusing the human attention in two ways: whilst annotated signal elements point out "recognizable" events, and submit them to further examination, non annotated events conversely either reveal a lack of knowledge, or suggest further exploration of the data.

To be noticed is the fact that any result, newly obtained at the segmentation level, is transmitted forward to the classification agent, which may in turn drive a further launching of segmentation agents. This process continues until some stabilization is being reached, which is controlled by human interventions.

Symbolic Translation Triad. The role of the symbolic translation triadic agent is to translate numerical time series into symbolic time series. It collects to this end the segments and models of segments computed by the segmentation and classification agents. For each segment $(S)$, the triadic agent creates a timestamped symbol with the date and duration of $S$ and the symbolic name $(N)$ of the most accurate models of segments. Concatenated time-stamped symbols for a given time series constitute the symbolic time series of a patient record. The 
whole process results in concatenated time-stamped symbols which characterize the patient record at the symbolic level.

For multivariate time series, one classification agent is dedicated to each type of time series (Flow, Paw, ...), and defines the corresponding segmentation agents. The symbolic time series merges time-stamped symbols from all types of time series.

Scenario Agents. The scenario construction is considered as the process of finding temporal relations between symbols. This process is driven by the assumption of some symbols, selected by the clinician, that are to be "explained" through their causal links to some ancestor "events" (symbols). The task of the scenario agent, associated to a symbol $E$, is to collect the symbols preceding each occurrence of $E$ in a temporal window with a fixed length. Each set of such symbols is then considered as an example for the learning process at hand. From these examples, the agent finally constructs the time-stamped pattern, i.e. the scenario that "explains" $E$. To this end, we use an extended version of the A Priori algorithm [14, which builds the biggest frequent time-stamped pattern. The resulting scenario may of course be submitted to the user, which may result in modifications in the proposed pattern, or collecting other example patterns to support or contradict the current interpretation. In a complementary feedback loop (link 4 in Figure 1), the proposed scenario may be considered as a model to drive further analysis. Any deviation from the current model is considered as a potential for improvement of the annotation process. The system will then focus its attention on the corresponding deviation locations.

\section{Patient-Ventilator Asynchronies Exploration Results}

\subsection{Rationale}

Patients suffering from respiratory disorders and hospitalized in Intensive Care Units are mechanically supported with assist-control ventilation. An adequate synchronization between patient and ventilator is then required to improve patient's comfort and optimize work of breathing. A recent study [1] shows that $24 \%$ of 60 patients mechanically ventilated exhibited an asynchrony index higher than $10 \%$ of respiratory efforts. Ineffective triggering, i.e. when patient's efforts do not result in ventilator triggering, was the major cause $(85 \%)$ of asynchrony. Asynchrony was associated with a longer duration of mechanical ventilation. Therefore, it is important to identify factors increasing the incidence of asynchrony. Some major asynchronies can be detected by an experienced clinician. This remains a difficult extra workload and no physiological models exist yet to allow for their automatic detection. In order to detect, from the physiological data, specific patterns, regularities or sequences of events (scenarios), associated to the occurrence of asynchrony, we provided a clinician with our system to annotate and explore respiratory time series. They were constituted of flow and airway pressure (Paw) signals continuously recorded during 30 minutes and sampled at $200 \mathrm{~Hz}$ (see [1] for details). 


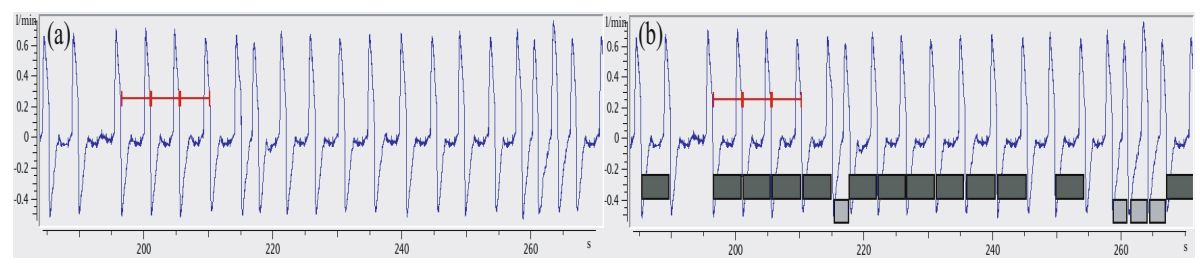

Fig. 2. Model construction (Flow signals). 2a (left): Two annotations (gray horizontal bar) are inserted on the flow signal by the clinician to indicate asynchrony periods. 2b (right): Annotation completion by the system: dark-gray boxes indicate retrieved asynchronies periods. Medium-gray boxes indicate retrieved non-asynchronies periods.

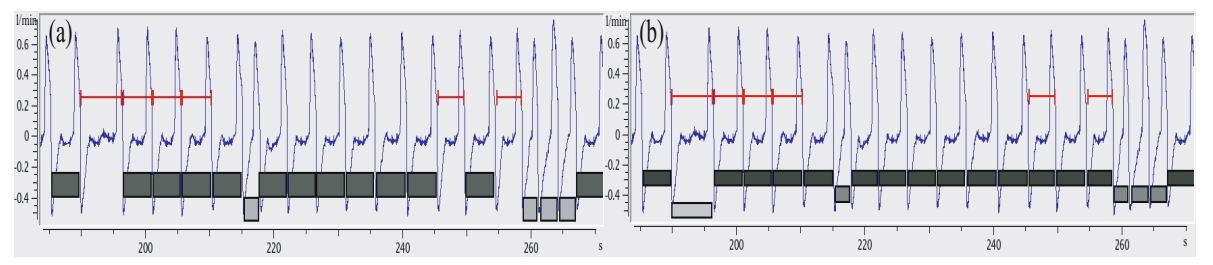

Fig. 3. Model discovery (Flow signals). 3a (left): The clinician has annotated the unrecognized segments. $3 \mathrm{~b}$ (right): A new model for double triggering periods has been discovered (single light-gray box), the "ineffective triggering" model has been refined to retrieve all ineffective triggering periods.

\subsection{Time Series Data Exploration Case Study}

We first of all illustrate the capacity of our system to build annotations in an autonomous way, thereby assisting the clinician in the elaboration of new interpretation models; we illustrate in a second step its capacity to validate previously constructed models, assisting the clinician in the model-driven investigation of unknown data. Alternating successive steps of model construction/model validation would finally allow the clinician to build a progressively refined interpretation of data, together with more robust models.

Models Construction and Automatic Asynchrony Detection. Three patient's records were fully interpreted via our system. Using the system interface, the clinician by a visual inspection, annotated some inefficient triggering (see Figure 2 2 ) on a specific part of the signal. Then, the clinician launched the processing step i.e. the execution of the classification agents, the segmentation agents and finally the symbolic translation triadic agent. Based on the initial partial annotation by the expert, the system symbolically labeled the complete time series (see Figure 2b). On the Figure2, all asynchronies considered as similar to those annotated by the clinician as ineffective triggering, were retrieved (dark-gray boxes). The three complete time series were automatically annotated based on clinician's annotations that represented about $30 \%$ of the total asynchronie events. The mean 
sensitivity and the mean specificity were respectively egal to 0.64 and 0.93 . Finally, the system constructed scenarios that bring new information about asynchrony occurrence conditions. For this purpose, Paw and Flow signals were automatically explored in a temporal windows (fixed to 10 s i.e. approximatively three periods) around asynchrony events. Our system detected that asynchrony on the Flow signal was associated in $90 \%$ of cases with asynchrony on the Paw signal and that they were preceeded by another asynchrony in $90 \%$ of cases. This extracted knowledge was then used to automatically detect deviant patterns, such as a single asynchrony (may be artefactual) or mismatch between flow and paw asynchronies. New annotations (artefacts, trends) should be inserted by the clinician to finalize the validation and enrich these first results.

Models Comparison and Extraction of New Patterns. Our system may also support the clinician in the model-driven investigation of unknown data, to validate the genericity of a previously constructed model. To illustrate this point, we used seven new data sets. For three of these data sets, the "ineffective triggering" model that was previously built from one patient data set, appeared clearly not relevant. However, for the four remaining data sets, the model revealed adapted and the clinician was able to identify new patterns: two ineffective triggering in the same period pattern (see Figure 3) and double triggering patterns. Extensive data exploration should be realized to confirm these preliminary results.

\section{Discussion and Perspectives}

We have presented an original approach to support clinicians in the difficult task of multivariate time series data interpretation. Our approach is centered on the collaboration between a clinician and an autonomous system, both embedded in a learning cycle. We advocate for the design of computerized tools that really support the clinician in his/her decision making process, rather than provide him/her with final results. The presented system participates to this medical computerized tools design evolution [8]. We are aware of the computing complexity of the processes we have presented. But we assume that data interpretation is a prospective task with no critical time constraints.

The undergoing experiments on patient ventilator asynchrony exploration strongly support the interest of our approach. We expect that the global system implementation in progress, including the feedback from scenario construction, will improve the detection of known events and consequently its sensitivity. Several extensions of the present implementation may be envisaged. The fusion of several physiological parameters would enable the construction of more elaborate asynchrony models, as well as taking into account contextual information such as pathology, treatments or the mode of ventilation used. The objective is to integrate contextual information on patient as a interpretation element and maybe enable clinician to construct typical scenarios for groups of patients. Finally, the preliminary experiments on scenario learning indicate that a more sophisticated 
time representation should be introduced (such as interval relations for example). Further experimentations in close collaboration with clinicians will allow us to fully evaluate the real impact of such as interactive approach for knowledge discovery and knowledge formalization.

Acknowledgments. We thank Dr. Thille and Pr. Brochard from H. Mondor Hospital (Créteil, Fr) for providing the clinical data and for comments.

\section{References}

1. Thille, A., Rodriguez, P., Cabello, B., Lellouche, F., Brochard, L.: PatientVentilator Asynchrony During Assisted Mechanical Ventilation. Intens. Care Med. 32(10), 1515-1522 (2006)

2. Salatian, A., Hunter, J.: Deriving Trends in Historical and Real-Time Continuously Sampled Medical Data. J. Intell. Inf. Syst. 13(1-2), 47-71 (1999)

3. Shahar, Y.: A Framework for Knowledge-Based Temporal Abstraction. Artif. Intell. 90(1-2), 79-133 (1997)

4. Augusto, J.C.: Temporal Reasonning for Decision Support in Medicine. Artif. Intell. Med. 33(2), 1-24 (2005)

5. Fromont, É., Quiniou, R., Cordier, M.O.: Learning Rules from Multisource Data for Cardiac Monitoring. In: Miksch, S., Hunter, J., Keravnou, E.T. (eds.) AIME 2005. LNCS (LNAI), vol. 3581, pp. 484-493. Springer, Heidelberg (2005)

6. Guimarães, G., Peter, J.H., Penzel, T., Ultsch, A.: A Method for Automated Temporal Knowledge Acquisition Applied to Sleep-Related Breathing Disorders. Artif. Intell. Med. 23(3), 211-237 (2001)

7. Silvent, A.S., Dojat, M., Garbay, C.: Multi-Level Temporal Abstraction for Medical Scenarios Construction. Int. J. Adapt. Control. 19(5), 377-394 (2005)

8. Zupan, B., Holmes, J., Bellazzi, R.: Knowledge Based Data Analysis and Interpretation. Artif. Intell. Med. 37(1), 163-165 (2006)

9. Kabanza, F., Bisson, G., Charneau, A., Jang, T.S.: Implementing Tutoring Strategies Into a Patient Simulator for Clinical Reasoning Learning. Artif. Intell. Med. 38(1), 79-96 (2006)

10. Lee, E., Chan, C., Aalst, J.: Students Assessing their Own Collaborative Knowledge Building. Int. J. of Computer-Supported Collaborative Learning 1(1), 57-87 (2006)

11. Morik, K., Imhoff, M., Brockhausen, P., Joachims, T., Gather, U.: Knowledge Discovery and Knowledge Validation in Intensive Care. Artif. Intell. Med. 19(3), 225-249 (2000)

12. Schroeder, L., Bazzan, A.: A Multi-Agent System to Facilitate Knowledge Discovery: An Application to Bioinformatics. In: Proceedings of the Workshop on Bioinformatics and Multiagent Systems (2002)

13. Bottoni, P., Garbay, C., Lecca, F., Mussio, P., Rizzo, P.: Collaborative Indexing and Retrieval by Annotation: the Case of Artistic Artifacts. In: Proceedings of the 2nd International Workshop on Content-based Multimedia Indexing, pp. 315-322 (2001)

14. Dousson, C., Duong, T.: Discovering Chronicles with Numerical Time Constraints from Alarm Logs for Monitoring Dynamic Systems. In: Dean, T. (ed.) Proceedings of the 16th International Joint Conference on Artificial Intelligence, pp. 620-626. Morgan Kaufmann, San Francisco (1999) 